\title{
Solving dynamic stochastic economic models by mathematical programming decomposition methods

\author{
Mercedes Esteban-Bravo ${ }^{\mathrm{a}, *}$, Francisco J. Nogales ${ }^{\mathrm{b}}$ \\ a Department of Business Administration, Universidad Carlos III de Madrid, C/Madrid 126, 28903 Getafe, Madrid, Spain
} \\ ${ }^{\mathrm{b}}$ Department of Statistics, Universidad Carlos III de Madrid, Avda. de la Universidad, 30. 28911 Leganés, Madrid, Spain
}

Available online 17 April 2006

\begin{abstract}
Discrete-time optimal control problems arise naturally in many economic problems. Despite the rapid growth in computing power and new developments in the literature, many economic problems are still quite challenging to solve. Economists are aware of the limitations of some of these approaches for solving these problems due to memory and computational requirements. However, many of the economic models present some special structure that can be exploited in an efficient manner. This paper introduces a decomposition methodology, based on a mathematical programming framework, to compute the equilibrium path in dynamic models by breaking the problem into a set of smaller independent subproblems. We study the performance of the method solving a set of dynamic stochastic economic models. The numerical results reveal that the proposed methodology is efficient in terms of computing time and accuracy.
\end{abstract}

Keywords: Dynamic stochastic economic model; Computation of equilibrium; Mathematical programming; Decomposition techniques

\section{Introduction}

In many situations, applied economists consider discrete-time stochastic dynamic models for forecasting, testing economic theories, and designing economic policies. These models have been extensively used in economics because this theoretical framework is flexible enough to represent the fluctuations of unemployment, prices, consumption, production and investment, among others. From a computational point of view, solving dynamic economic problems is still a quite challenging task, in spite of the noticeable increase in computing power, storage capacity and new approaches in the literature on computational economics. The search of major realism in the economic models have pushed economists to consider more and more complex dynamic stochastic specifications which generally challenge the best existing approaches. One of the fundamental difficulties with the solvability of many economic models is that the computational load grows with the dimension and complexity of the problem. Despite the fact that most of these approaches show good performance in solving particular economic models, a computational methodology to deflate the curse of dimensionality is worthwhile and would be appreciated by practitioners to enlarge substantially the class of questions that can be addressed with dynamic modelling. 
Many different algorithms have been proposed during the past years. There are two main approaches to approximate the solution of a dynamic economic problem: the discrete approximation and the smooth approximation. The first approach consists of discretizing the value of the policy function over a refined grid of points and then solving the Bellman operator for each point of the grid (see [1]). The second approach considers parametric approximations, based on Taylor series expansions, of the value of the policy function (see [2-5], among others). In many problems, the Euler equation (which represents the first-order conditions for the problem corresponding to the Bellman equation) is used to approximate the value function. The log polynomials approximations (see e.g., [6]), projection methods as introduced in [7] and perturbation methods (see e.g., [8,2]) are popular procedures for approximating the Euler equations. For a discussion and analysis of these approaches see [9]. More recently, in [10], Judd et al. review advances in computational methods for solving dynamic models and Borağan et al., in [11], study the performance and accuracy of different solution methods, making a clear recommendation of perturbation methods.

Other approaches involve converting the problem into a mathematical programming problem in which all the states and controls were decision variables and the dynamic equations formed part of the constraint set (see [12,13]). But these methods lead to problems with large numbers of variables and constraints. We aim to address this problem by splitting it into manageable pieces (subproblems) and by coordinating the solutions of these subproblems. To attain this goal, we have studied the special structure of economic dynamic stochastic models. Then, we have developed a general methodology based on a decomposition procedure to reduce the dimensionality problem associated with dynamic stochastic models. This methodology solves the original model by breaking the problem into a set of smaller independent problems. With the proposed methodology, we obtain two main computational advantages. First, the subproblems are, by definition, smaller than the original problem and therefore much faster to solve. Second, the subproblems could have special properties such as convexity and sparsity that enable the use of efficient algorithms to solve them.

Previous decomposition algorithms based on mathematical programming are categorized into three groups: DanztingWolfe decomposition, Benders decomposition and augmented Lagrangian relaxation procedures. Both Danzting-Wolfe decomposition (see [14]) and Benders decomposition (see [15,16]) are efficient schemes to deal with convex optimization problems. Extension to nonconvex problems is attained by the augmented Lagrangian relaxation (see [17-20]). These techniques are based on an estimate of the Lagrange multipliers to decompose the problem into a set of subproblems. Then, their solutions are used to update the current estimate of the Lagrange multipliers. But augmented Lagrangian methods may converge slowly in practice (see [21,22]).

Applications of decomposition methods to economics are originally due to Mansur and Whalley [23]. They apply Danzting and Wolfe's decomposition to compute the solution of a pure exchange general equilibrium model. A pure exchange general equilibrium problem represents a static economy where there are no sectors of production (for a detail description of the model see [24]). In contrast, the current paper considers an extension of Lagrangian decomposition methods for the computation of stochastic dynamic economic models. It must be noted that these models represent a more general and versatile tool in economics as they describe an economy with consumption and production sectors that evolves over an infinite number of time periods. Our approach first reduces the original problem to a finite-horizon problem and then solves decomposed subproblems obtained after fixing some of the decision variables and Lagrange multipliers.

To validate the efficiency of the proposed methodology, we have solved several economic dynamic models. The numerical results are very encouraging, showing computational gains when applying to large-scale problems. The proposed approach has therefore the potential for application in many economics problems.

The paper proceeds as follows. In Section 2, for illustrative purposes, we consider a simple but important example, the traditional deterministic neoclassical growth model. We use this model to develop, in Section 3, the proposed decomposition methodology. In Section 4, we extend this methodology for dealing with uncertainty. In Section 5 we present and solve an international model which is typically hard to solve because of its high dimensionality. Finally, in Section 6, we discuss the results and provide conclusions.

\section{The economic growth model}

The neoclassical growth model, despite its age and recent developments in the growth literature, is widely used by academics because of its great theoretical and empirical interest to study the long-term economic performance (see e.g. 
$[25,26])$. In these models, agents have to decide (in each period) how to allocate their resources between consumption commodities, in order to provide instantaneous utility and capital commodities, and obtaining production for the next period. Therefore, these models are usually used for forecasting, testing economic theories, and designing economic policies that increase the growth rate in the long-term. For a formal discussion of these models, see e.g., [27].

The neoclassical growth model can be motivated as follows. Suppose a dynamic economy with infinitely lived agents, whose preferences are representable by the utility function $U(c)=\sum_{t=0}^{\infty} \beta^{t} u\left(c_{t}\right)$, where $\left\{c_{t}\right\}$ is the sequence of consumption at each period $t, u\left(c_{t}\right)$ is the utility function at each date and $\beta \in(0,1)$ is the discount factor. It is assumed that the agents aim at maximizing their present utility represented by $U(c)$, and that $U(c)$ is strictly increasing and strictly concave $C^{2}$ function. The physical capital is assumed to evolve according the law of motion $k_{t+1}=F\left(k_{t}\right)-c_{t}$, for $t=0,1, \ldots$, and where $F\left(k_{t}\right)$ denotes a production function, given an initial endowment $k_{0}=\bar{k}_{0}>0$ of the capital stock. It is assumed that $F\left(k_{t}\right)$ is a strictly increasing and strictly concave $C^{2}$ function.

Modern economic theory is based upon the concept of competitive equilibrium, which consists of an array of prices and allocations (the consumption and the physical capital decisions) equating aggregate supply and demand. Equilibrium is a basic descriptive and predictive tool for economists because it is expected that the forces acting on the economy will drive to this array of allocations and prices. However, we can assume that both the consumption and the physical capital decision are made by a representative agent, the social planner. In welfare economics, a social planner is a decision-maker who attempts to achieve Pareto optimality, in which no one's outcome can be improved without worsening someone else's outcome. The social planner role can be thought of as if it were played by a government. Two important results in economics, called the Two Fundamental Theorems of Welfare Economics, link the concept of a Pareto-optimal allocation with that of a competitive equilibrium under certain conditions. See [28,27] for details. Since both welfare theorems hold in this economy, our problem reduces to solve the social's planner problem (i.e. to solve a Pareto-optimal allocation):

$$
\begin{aligned}
\max & \sum_{t=0}^{\infty} \beta^{t} u\left(c_{t}\right) \\
\text { s.t. } & k_{t+1}-F\left(k_{t}\right)+c_{t}=0, \quad t=0,1, \ldots,
\end{aligned}
$$

given an initial condition $k_{0}=\bar{k}_{0}$. The social planner problem is usually easier to solve than the equilibrium problems.

\section{The decomposition methodology}

In this section, we describe a general decomposition methodology to compute economic equilibria in dynamic models. Decomposition is a classical solution approach for optimization problems based on the idea of partition. To simplify our exposition, we consider the standard deterministic neoclassical growth model (1), which encompasses most of discrete-time models proposed in the economic literature.

The first step of the proposed decomposition procedure follows the conventional domain truncation technique. Mainly, we consider a large but finite temporal horizon of problem (1), $0<T<\infty$. Therefore, the truncated problem has the form

$$
\begin{aligned}
\max & \sum_{t=0}^{T} \beta^{t} u\left(c_{t}\right) \\
\text { s.t. } & k_{t+1}-F\left(k_{t}\right)+c_{t}=0, \quad t=0, \ldots, T, \\
& k_{0}=\bar{k}_{0} .
\end{aligned}
$$

Under our assumptions regarding preferences and technology, a solution of problem (1) is characterized by the Euler equation and the transition law for the capital stock

$$
\begin{aligned}
& u^{\prime}\left(c_{t}\right)=\beta u^{\prime}\left(c_{t+1}\right) F^{\prime}\left(k_{t+1}\right), \\
& k_{t+1}-F\left(k_{t}\right)+c_{t}=0 \text { for all } t=0,1, \ldots .
\end{aligned}
$$


SP0. For $t=0$ :

$$
\begin{aligned}
& \max \beta^{0} u\left(c_{0}\right) \\
& \text { subject to } \bar{k}_{1}-F\left(\bar{k}_{0}\right)+c_{0}=0
\end{aligned}
$$

(with $\bar{k}_{1}$-fixed from previous iteration);

SPt. For $t=1, \ldots, T-1$ :

$$
\begin{aligned}
& \max \beta^{t} u\left(c_{t}\right)-\bar{\lambda}_{t-1}\left(k_{t}-F\left(\bar{k}_{t-1}\right)+\bar{c}_{t-1}\right) \\
& \text { subject to } \bar{k}_{t+1}-F\left(k_{t}\right)+c_{t}=0
\end{aligned}
$$

(with $\bar{\lambda}_{t-1}, \bar{k}_{t-1}, \bar{k}_{t+1}$ and $\bar{c}_{t-1}-$ fixed from previous iteration);

SPT. For $t=T$ :

$$
\begin{aligned}
& \max \beta^{T} u\left(c_{T}\right)-\bar{\lambda}_{T-1}\left(k_{T}-F\left(\bar{k}_{T-1}\right)+\bar{c}_{T-1}\right)+\epsilon_{T} \ln \left(\bar{k}_{T+1}\right) \\
& \text { subject to } \bar{k}_{T+1}-F\left(k_{T}\right)+c_{T}=0,
\end{aligned}
$$

and compute $\bar{k}_{T+1}=F\left(k_{T}\right)-c_{T}$ (with $\bar{\lambda}_{T-1}, \bar{k}_{T-1}, \bar{k}_{T+1}$ and $\bar{c}_{T-1}-$ fixed from previous iteration).

Fig. 1. Basic iteration of the decomposition algorithm.

To find an optimum, we need the transversality condition (some sort of second-order condition relating to the concavity of the objective function at the candidate point). The transversality condition associated with problem (1) is given by

$$
\lim _{T \rightarrow \infty} \lambda_{T} k_{T+1}=0
$$

where $\lambda_{t}$ denotes the Lagrange multiplier associated to the $t$ th constraint: $k_{t+1}-F\left(k_{t}\right)+c_{t}=0$. To accommodate the transversality condition, we propose to add a barrier term to the capital stock at $T+1$, guaranteeing that an optimal solution for problem (1) is a solution of the following problem:

$$
\begin{aligned}
\max & \sum_{t=0}^{T} \beta^{t} u\left(c_{t}\right)+\varepsilon_{T} \ln \left(k_{T+1}\right) \\
\text { s.t. } & k_{t+1}-F\left(k_{t}\right)+c_{t}=0, \quad t=0, \ldots, T, \\
& k_{0}=\bar{k}_{0},
\end{aligned}
$$

where $\varepsilon_{T}>0$ and $\lim _{T \rightarrow \infty} \varepsilon_{T}=0$. An optimum of problem (4) satisfies

$$
\begin{aligned}
& u^{\prime}\left(c_{t}\right)=\beta u^{\prime}\left(c_{t+1}\right) F^{\prime}\left(k_{t+1}\right), \\
& k_{t+1}-F\left(k_{t}\right)+c_{t}=0 \text { for all } t=0, \ldots, T, \\
& \varepsilon_{T}=\lambda_{T} k_{T+1} .
\end{aligned}
$$

Observe that the resulting optimality conditions coincide with those of problem (1) for the optimal values $\left(c^{*}, k^{*}\right)$ in the limit $T \rightarrow \infty$ as $\lim _{T \rightarrow \infty} \varepsilon_{T}=0$. However, these truncated problems can be too large to be solved by standard algorithms. For this reason, we propose to use the following decomposition approach which alleviates the high dimensionality by breaking problem (4) into a set of smaller independent subproblems. The separability of the subproblems is obtained by fixing the values of some variables.

At each iteration of the procedure, the following $T+1$ subproblems (SP) are solved (Fig. 1). 
Initialization: Set a truncation date $T$ and a regularization parameter $\epsilon_{T}$. Choose a starting point $\left(\bar{c}_{0}, \bar{c}_{1}, \ldots, \bar{c}_{T}, \bar{k}_{1}, \ldots, \bar{k}_{T+1}\right)$ and an initial set of multipliers $\left(\bar{\lambda}_{0}, \bar{\lambda}_{1}, \ldots, \bar{\lambda}_{T}\right)$. Set $k \leftarrow 0$.

\section{Repeat:}

1. Solve subproblems SPt in $c_{t}$ and $k_{t}$ for all $t=0,1, \ldots, T$. Denote by $\left(\widetilde{c}_{0}, \widetilde{c}_{1}, \ldots, \widetilde{c}_{T}, \widetilde{k}_{1}, \ldots, \widetilde{k}_{T^{\prime}+1}\right)$ the solution of these subproblems for all $t=0,1, \ldots, T$ and $\left(\widetilde{\lambda}_{0}, \widetilde{\lambda}_{1}, \ldots, \widetilde{\lambda}_{T}\right)$ the associated optimal multipliers.

2. Update new point and multipliers:

$$
\begin{aligned}
\left(\bar{c}_{0}, \bar{c}_{1}, \ldots, \bar{c}_{T}, \bar{k}_{1}, \ldots, \bar{k}_{T+1}\right) & \leftarrow\left(\widetilde{c}_{0}, \widetilde{c}_{1}, \ldots, \widetilde{c}_{T}, \widetilde{k}_{1}, \ldots, \widetilde{k}_{T+1}\right) \\
\left(\bar{\lambda}_{0}, \bar{\lambda}_{1}, \ldots, \bar{\lambda}_{T}\right) & \leftarrow\left(\widetilde{\lambda}_{0}, \widetilde{\lambda}_{1}, \ldots, \widetilde{\lambda}_{T}\right)
\end{aligned}
$$

and set $k \leftarrow k+1$.

\section{Until convergence (condition (5) is satisfied):}

Fig. 2. Decomposition algorithm.

The intuitive idea of this decomposition methodology is to consider a set of smaller independent problems whose first-order necessary conditions (at the optimal the solution) coincide with the corresponding conditions for problem (4). For each subproblem $t$, the decision variables are only the contemporary ones (for example, the subproblem SPt is solved in $c_{t}$ and $k_{t}$ ). An economic interpretation of the decomposition draws on this partition of the decision variables into contemporary and non contemporary decisions taken among agents.

Once the solutions for these subproblems have been computed, the multipliers and the fixed variables are updated. If a parallel implementation is required, then these values are updated from previous iteration. If a sequential implementation is required, then these values are updated according to the most recently computed values (the corresponding to the last subproblem solved).

This procedure is repeated until the convergence criteria for the global finite problem (4) are satisfied. We have chosen the following stopping criterion (where the superscript ${ }^{l}$ denotes the current iteration):

$$
\frac{\left\|L\left(c^{l}, k^{l}\right)-L\left(c^{l-1}, k^{l-1}\right)\right\|}{1+\left\|L\left(c^{l-1}, k^{l-1}\right)\right\|} \leqslant \varepsilon,
$$

where $L\left(c^{l}, k^{l}\right)=\sum_{t=0}^{T} \beta^{t} u\left(c_{t}^{l}\right)+\varepsilon_{T} \ln \left(k_{T+1}^{l}\right)$ denotes the value of the objective function at iteration $l$.

The scheme of the decomposition algorithm is stated as in Fig. 2.

Following [20], the convergence properties of the proposed algorithm depend on the spectral radius of a matrix related to the Newton matrix of the global problem. If this spectral radius is smaller than one, local convergence is guaranteed. This condition is problem dependent but in many practical cases this condition is satisfied. However, if this condition does not hold, local convergence can be guaranteed modifying the proposed methodology by using preconditioned Conjugate Gradient techniques, as explained in [20]. The decomposition algorithm converges in all the studied cases without the need of using Conjugate Gradient techniques in the linear algebra level. Further, the convergence properties of this algorithm do not require an optimal solution of subproblems SP0, SPt and SPT. It is enough to compute their solution up to a certain degree of accuracy (near the solution, it could be enough to perform a single iteration for each subproblem). Therefore, the proposed decomposition technique considerably increases the speed of computations.

Next a numerical experiment is introduced to illustrate the computational gains of the decomposition method. We have considered the neoclassical growth model presented in Section 2. In this model, we have chosen a Cobb-Douglas 
Table 1

Running times (in seconds) until convergence for different $T$ 's

\begin{tabular}{|c|c|c|c|c|c|}
\hline$T$ & Direct & Decomposition & $T$ & Direct & Decomposition \\
\hline 75 & 1.0 & 2.7 & 775 & 121.4 & 23.0 \\
\hline 125 & 2.1 & 3.8 & 825 & 126.3 & 25.8 \\
\hline 175 & 4.3 & 5.4 & 875 & 200.0 & 27.4 \\
\hline 225 & 7.1 & 6.5 & 925 & 188.4 & 27.5 \\
\hline 275 & 9.3 & 8.7 & 975 & 278.5 & 28.01 \\
\hline 325 & 11.5 & 9.8 & 1000 & 279.6 & 29.6 \\
\hline 375 & 17.5 & 10.9 & 1050 & 309.3 & 30.2 \\
\hline 425 & 23.0 & 12.0 & 1100 & 350.5 & 32.4 \\
\hline 475 & 32.4 & 15.3 & 1150 & 702.0 & 46.1 \\
\hline 525 & 43.9 & 14.2 & 1200 & 828.4 & 38.4 \\
\hline 575 & 50.5 & 18.1 & 1250 & 848.1 & 46.1 \\
\hline 625 & 65.3 & 21.4 & 1300 & 1174.4 & 39.0 \\
\hline 675 & 74.7 & 21.5 & 1350 & 1799.0 & 46.6 \\
\hline 725 & 88.4 & 22.5 & & & \\
\hline
\end{tabular}

production function $F(k)=k^{\alpha}$ with capital share $\alpha=0.33$, and a utility function $u(c)=c^{\rho} / \rho$ with $\rho=0.4$. The discount factor is $\beta=0.8$ and the regularization parameter has been fixed to $\varepsilon_{T}=10^{-4}$.

We have implemented the direct and the decomposition algorithm using MATLAB 7.0 on an Intel Pentium IV $1.7 \mathrm{GHz}$ with machine precision $10^{-16}$. The solution of neoclassical growth model and the corresponding subproblems SPt, for $t=0, \ldots, T$, have been solved using TOMLAB/KNITRO (v. 4.0). KNITRO implements state-of-the-art methods for solving nonlinear optimization problems. We have selected an active-set sequential linear-quadratic method for KNITRO. The reason is that, for the decomposition algorithm, this method is preferable (compared to an interior-point method) because good initial points can be provided (from previous iterations).

The decomposition algorithm stops whenever $\varepsilon=10^{-8}$. Table 1 reports a comparison of the running times (in seconds) until convergence obtained from both procedures.

We can see a better view of these running times (in logarithmic scale) in Fig. 3.

From this figure, it can be shown a clear advantage of the proposed decomposition algorithm over the direct approach. It is remarkable that the computing time until convergence (or curse of dimensionality) required to solve the original problem (2) is much better using the decomposition methodology than using a direct approach. Table 1 shows that the proposed methodology is an effective and useful tool for solving large-scale economic problems as it breaks down a high-dimensional problem into many low-dimensional ones, hence reducing the curse of dimensionality. Note that the proposed decomposition algorithm exploits the structure of some optimization problems by reformulating them as a set of independent subproblems, in our case one per period. Then, the Lagrange multipliers computed from the subproblems are used to coordinate the subproblem solutions and find an overall problem solution. Of course, the rate of convergence of the decomposition algorithm will be slower than that of a direct algorithm. But the cost per iteration will be much better due to the size (small) of the subproblems (and the good starting points obtained in previous iterations). This is the reason why, in these cases, the decomposition approach is faster than direct approaches.

Moreover, to make the decomposition algorithm comparable to standard approaches in computational economics literature, we consider the accuracy of the solution measured by the normalized Euler equation error over $T$ periods

$$
E E_{N}=\max _{t=0,1, \ldots, T} \frac{\left|E_{t}\right|}{u^{\prime}\left(c_{t}\right) c_{t}},
$$

where $E_{t}$ represents, in some sense, the first-order necessary conditions for problem (1). The use of Euler equation error is a common and reliable feature of most traditional approaches (see [7]). In our example,

$$
E_{t}=c_{t}^{\rho-1}-\beta \alpha c_{t+1}^{\rho-1} k_{t}^{\alpha-1} \quad \text { for all } t=0,1, \ldots, T .
$$

Judd and Guu, in [8], interpret this error as the relative optimization error incurred by the use of the approximated policy rule. Our algorithm achieves a good approximation of the solution of model (1) as the maximum Euler error is $6.57 \times 10^{-7}$ for $T=1350$. This means that the agent is making a cent mistake for each $\$ 10^{6}$ of dollars spent. 


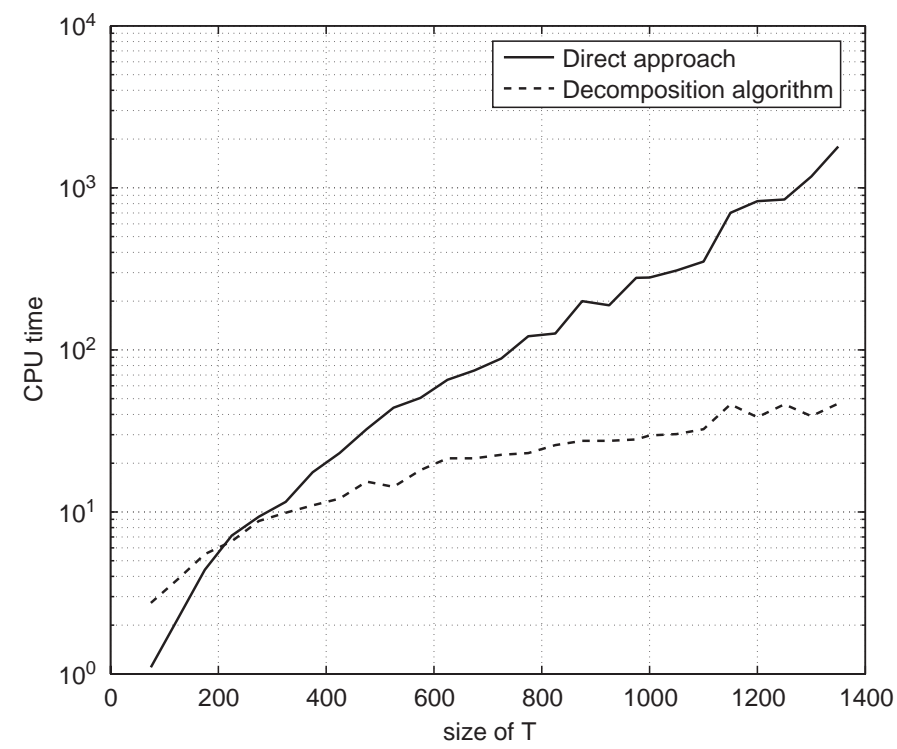

Fig. 3. Running times (in logarithmic scale) until convergence for different $T$ 's.

This accuracy is comparable to that reported by Judd in [7], taking into account that we have set the termination tolerance for the subproblems as $\varepsilon=10^{-8}$.

Though the results are very encouraging, in terms of computing time and accuracy of the solution, this example is too simple to draw any firm conclusion. The next sections include models with uncertainty in some parameters and which are more realistic from a practical point of view.

\section{Solving stochastic growth models}

In macroeconomics, it is well-known that it is essential to incorporate uncertainty into the decision-making problems. The resulting stochastic models provide a tighter link between the specification of the economic theory and the empirical facts. In this section, we show how to extend the proposed decomposition methodology presented in Section 3 to solve stochastic dynamic economic problems.

We present the decomposition algorithm in the context of the following stochastic optimal growth problem:

$$
\begin{aligned}
\max & E\left[\sum_{t=0}^{\infty} \beta^{t} u\left(c_{t}\right)\right] \\
\text { s.t. } & k_{t+1}-F\left(k_{t}, \theta_{t}\right)+c_{t}=0, \quad t=0, \ldots, \infty, \\
& k_{0}=\bar{k}_{0},
\end{aligned}
$$

where $\theta_{t}$ denotes the stochastic shock to technology at time $t$.

After choosing $T$, the truncated problem is defined as follows:

$$
\begin{aligned}
\max & E\left[\sum_{t=0}^{T} \beta^{t} u\left(c_{t}\right)+\varepsilon_{T} \ln \left(k_{T+1}\right)\right] \\
\text { s.t. } & k_{t+1}-F\left(k_{t}, \theta_{t}\right)+c_{t}=0, \quad t=0, \ldots, T,
\end{aligned}
$$


where $k_{0}=\bar{k}_{0}, \varepsilon_{T}>0$ and $\lim _{T \rightarrow \infty} \varepsilon_{T}=0$. Then, the decomposition procedure to solve (9) is similar to the deterministic case after representing uncertainties in a form suitable for computation.

As we are using a mathematical programming framework, the uncertainty presented in these problems must be represented in such a manner that its effect on present decision-making can properly be taken into account. A common representation of the uncertainty is to work with scenarios, which are particular models of how the future might unfold. Within this framework, simulations can be used to generate a batch of scenarios.

Moreover, for the class of problems studied in this paper, agents are assumed to act with perfect foresight, that is, the decisions are made in the initial time period and then just watch the future unfold. This is justified assuming that the market is complete (that is, a complete set of possible gambles on future states-of-the-world can be constructed with existing assets). Completeness is a common assumption in Economics, where the set of states-of-the-world is formally defined (see e.g., $[25,26])$. Assuming that the market is complete, a perfect foresight equilibrium can be reinterpreted as a sequential market equilibrium (agents are required to take decisions every period). This is due to the Equivalence Theorem (see [28, Proposition 19.D.1]).

For this reason, stochastic growth models can be decomposed by scenarios and hence, we only need to solve an optimization problem for each scenario that is generated. In model (9), uncertainty is represented by a scenario tree $S$. Final nodes $s$ represent the possible states realized by time $t$, that is, the complete history of exogenous aggregate shocks. The path to event $s$ is a partial scenario with probability $\omega_{s}$ along the path (see e.g., [29] for further details on this terminology).

Hence, problem (9) can be written by the following deterministic equivalent formulation

$$
\begin{aligned}
\max & \sum_{s \in S} \omega_{s}\left(\sum_{t=0}^{T} \beta^{t} u\left(c_{t, s}\right)+\varepsilon_{T} \ln \left(k_{T+1, s}\right)\right) \\
\text { s.t. } & k_{t+1, s}-F\left(k_{t, s}, \theta_{t, s}\right)+c_{t, s}=0, \quad t=0, \ldots, T, s \in S .
\end{aligned}
$$

As commented before, for each scenario $s \in S$, problem (10) can be broken into a set of independent problems of the form

$$
\begin{aligned}
\max & \sum_{t=0}^{T} \beta^{t} u\left(c_{t, s}\right)+\varepsilon_{T} \ln \left(k_{T+1, s}\right) \\
\text { s.t. } & k_{t+1, s}-F\left(k_{t, s}, \theta_{t, s}\right)+c_{t, s}=0, \quad t=0, \ldots, T,
\end{aligned}
$$

that can be broken into another set of independent problems for each period of time $t$, similarly to problem (4).

Therefore, we decompose the stochastic dynamic problem by scenarios and by time, obtaining the solution path $\left\{c_{t, s}, k_{t, s}\right\}_{t}$ for each scenario $s \in S$. At each iteration of the procedure, the following $|S|(T+1)$ subproblems are solved (Fig. 4).

With this approach, the expected values of $\left\{c_{t, s}, k_{t, s}\right\}_{t}$ will be consistent estimates of the expected value of the solution path, that is,

$$
E\left[c_{t}^{*}\right]=\sum_{s \in S} \omega_{s} c_{t, s}, \quad E\left[k_{t}^{*}\right]=\sum_{s \in S} \omega_{s} k_{t, s} \text { for all } t=0,1, \ldots, T,
$$

(where* denotes optimal solution) and the optimal value function can be estimated by means of

$$
E\left[V^{*}\right]=\sum_{t=0}^{T} \beta^{t} \sum_{s \in S} \omega_{s} u\left(c_{t, s}\right) .
$$

In general, scenario specifications of stochastic problems can be easily formulated but not solved. In applications we confine ourselves with some approximate distributions of random data comprising only finitely many scenarios. In other words, we assume $S$ is a finite set, $S=\{1, \ldots,|S|\}$.

The proper selection of the size $|S|$ is problem dependent. As a general rule, if $V_{|S|}^{*}$ represents an estimation of $E\left[V^{*}\right]$ for a given size $|S|$, we propose to try several values of $|S|$ until the estimation $V_{|S|}^{*}$ is stabilized. That is, we can choose 
SP0. For $t=0$ :

$$
\begin{aligned}
& \max \beta^{0} u\left(c_{0, s}\right) \\
& \text { subject to } \quad \bar{k}_{1, s}-F\left(\bar{k}_{0, s}, \theta_{0, s}\right)+c_{0, s}=0,
\end{aligned}
$$

(with $\bar{k}_{1, s}$-fixed from previous iteration);

SPt. For $t=1, \ldots, T-1$ :

$$
\begin{aligned}
& \max \beta^{t} u\left(c_{t, s}\right)-\bar{\lambda}_{t-1, s}\left(k_{t, s}-F\left(\bar{k}_{t-1, s}, \theta_{t-1, s}\right)+\bar{c}_{t-1, s}\right) \\
& \text { subject to } \bar{k}_{t+1, s}-F\left(k_{t, s}, \theta_{t, s}\right)+c_{t, s}=0,
\end{aligned}
$$

(with $\bar{\lambda}_{t-1, s}, \bar{k}_{t-1, s}, \bar{k}_{t+1, s}$ and $\bar{c}_{t-1, s}$-fixed from previous iteration);

SPT. For $t=T$ :

$$
\begin{aligned}
& \max \quad \beta^{T} u\left(c_{T, s}\right)-\bar{\lambda}_{T-1, s}\left(k_{T, s}-F\left(\bar{k}_{T-1, s}, \theta_{T-1, s}\right)+\bar{c}_{T-1,}\right)+\epsilon_{T} \ln \left(\bar{k}_{T+1, s}\right) \\
& \text { subject to } \bar{k}_{T+1, s}-F\left(k_{T, s}, \theta_{T, s}\right)+c_{T, s}=0,
\end{aligned}
$$

and compute $\bar{k}_{T+1, s}=F\left(k_{T, s}, \theta_{T, s}\right)-c_{T, s}\left(\right.$ with $\bar{\lambda}_{T-1, s}, \bar{k}_{T-1, s}, \bar{k}_{T+1, s}$ and $\bar{c}_{T-1, s}$-fixed from previous iteration).

Fig. 4. Basic iteration of the decomposition algorithm in the stochastic context.

$\left|S^{\prime}\right|>|S|$ until

$$
\frac{\left|V_{|S|}^{*}-V_{\left|S^{\prime}\right|}^{*}\right|}{\left|V_{|S|}^{*}\right|}<\varepsilon
$$

where for instance $\varepsilon=0.01$ represents a $1 \%$ of error.

Once the set $S$ is fixed, we compute the approximate solution $\left\{c_{t, s}^{*}, k_{t, s}^{*}\right\}_{t}$ for each $s \in\{1, \ldots,|S|\}$ and report the main statistical properties of the approximation. For example, the mean and variance of the solution path can be computed as follows:

$$
\begin{aligned}
& \widehat{E}\left[c_{t}^{*}\right]=\frac{1}{|S|} \sum_{s=1}^{|S|} c_{t, s}^{*}, \quad \widehat{E}\left[k_{t}^{*}\right]=\frac{1}{|S|} \sum_{s=1}^{|S|} k_{t, s}^{*} \text { for all } t=0,1, \ldots, T, \\
& \widehat{V}\left[c_{t}^{*}\right]=\frac{1}{|S|} \sum_{s=1}^{|S|}\left(c_{t, s}^{*}-\widehat{E}\left[c_{t}^{*}\right]\right)^{2}, \quad \widehat{V}\left[k_{t}^{*}\right]=\frac{1}{|S|} \sum_{s=1}^{|S|}\left(k_{t, s}^{*}-\widehat{E}\left[k_{t}^{*}\right]\right)^{2}, \text { for all } t=0,1, \ldots, T .
\end{aligned}
$$

Note that random sampling from a probability distribution ensures an essentially perfect (the accuracy is set by the researcher) knowledge of the distribution of any statistics based on the probability driving the sample variability. Note that we do not require stationarity as we estimate the statistical properties of the solution process from independent simulations. The Law of Large Numbers and Central Limit Theorems can be applied when the standard finite moments assumptions hold. In particular, if we generate independent realizations $s=1, \ldots,|S|$, then by the Kolmogorov Strong Law of Large Numbers, for any measurable function $g$ such that $E\left[\left|g\left(c_{t, s}^{*}\right)\right|\right]<\infty, E\left[\left|g\left(k_{t, s}^{*}\right)\right|\right]<\infty$ then

$$
\widehat{E}\left[g\left(c_{t, s}^{*}\right)\right]=\frac{1}{|S|} \sum_{s=1}^{|S|} g\left(c_{t, s}^{*}\right) \longrightarrow \text { a.s. } E\left[g\left(c_{t, s}\right)\right],
$$




\begin{tabular}{|c|c|c|c|c|c|c|}
\hline & \multicolumn{3}{|l|}{$T=50$} & \multicolumn{3}{|l|}{$T=100$} \\
\hline & $|S|=25$ & $|S|=50$ & $|S|=100$ & $|S|=25$ & $|S|=50$ & $|S|=100$ \\
\hline$\widehat{E}\left[V^{*}\right]$ & 66.00 & 65.66 & 65.97 & 104.87 & 106.08 & 106.85 \\
\hline Standard error of $\widehat{E}\left[V^{*}\right]$ & 0.21 & 0.15 & 0.11 & 0.25 & 0.18 & 0.12 \\
\hline Confidence Interval $(95 \%)$ of $E\left[V^{*}\right]$ & {$[65.58,66.41]$} & {$[65.36,65.95]$} & {$[65.75,66.18]$} & {$[104.38,105.36]$} & {$[105.72,106.43]$} & {$[106.61,107.08]$} \\
\hline CPU Time (seconds) & 132.8 & 235.1 & 497.2 & 290.4 & 542.6 & 1210.0 \\
\hline
\end{tabular}

$$
\widehat{E}\left[g\left(k_{t, s}^{*}\right)\right]=\frac{1}{|S|} \sum_{s=1}^{|S|} g\left(k_{t, s}^{*}\right) \longrightarrow \text { a.s. } E\left[g\left(k_{t, s}\right)\right] .
$$

This argument can also be applied to the variances as follows,

$$
\begin{aligned}
& \widehat{V}\left[c_{t, s}^{*}\right]=\frac{1}{|S|} \sum_{s=1}^{|S|}\left(c_{t, s}^{*}-\widehat{E}\left[c_{t, s}^{*}\right]\right)^{2} \longrightarrow \text { a.s. } V\left[c_{t, s}\right], \\
& \widehat{V}\left[k_{t, s}^{*}\right]=\frac{1}{|S|} \sum_{s=1}^{|S|}\left(k_{t, s}^{*}-\widehat{E}\left[k_{t, s}^{*}\right]\right)^{2} \longrightarrow \text { a.s. } V\left[k_{t, s}\right] .
\end{aligned}
$$

In addition, by the Lindeberg-Lèvy Central Limit Theorem, if $\left\{k_{t, s}^{*}\right\}$ are identically and independently distributed random variables with mean $E\left[k_{t, s}\right]$ and variance $V\left[k_{t, s}\right]<\infty$, then

$$
\frac{1}{\sqrt{|S|}} \sum_{s=1}^{|S|} \frac{\left(k_{t, s}^{*}-E\left[k_{t, s}\right]\right)}{\sqrt{V\left[k_{t, s}\right]}} \longrightarrow_{\mathrm{d}} N(0,1) .
$$

The same argument can be applied to any variable in the model.

Confidence intervals can be given by applying standard statistical methods. For example, for large $|S|$, we can compute a confidence interval (with probability $1-\alpha$ ) for the expected value of the consumption at time $t$ as

$$
E\left[c_{t}^{*}\right] \in \widehat{E}\left[c_{t}^{*}\right] \pm z_{\alpha / 2} \sqrt{\frac{\widehat{V}\left[c_{t}^{*}\right]}{|S|}},
$$

where $z_{\alpha / 2}$ is the appropriate percentile of the standard normal distribution. Analogously, the confidence intervals for other estimations can be established. Moreover, as in Section 3, we consider the accuracy of the solution measured by the normalized Euler equation error over $T$ periods (with its corresponding confidence interval).

Next, we present the results obtained by solving different cases of model (8) using the proposed decomposition algorithm. The solution of the stochastic growth subproblems SPt, for $t=0, \ldots, T$, have been solved using KNITRO with an active-set sequential linear-quadratic method.

We have considered a Cobb-Douglas production function $F\left(k_{t}, \theta_{t}\right)=\theta_{t} k_{t}^{\alpha}$ with capital share $\alpha=0.33$, and a utility function $c^{\rho} / \rho$ with $\rho=0.4$. The discount factor is $\beta=0.99$ and the regularization parameter has been set to $\varepsilon_{T}=10^{-4}$. The shocks $\left\{\theta_{t}\right\}$ are assumed to take the values $\{0.9,1,1.1\}$, following a first-order Markov chain given by the following transition matrix:

$$
\pi=\left(\begin{array}{ccc}
0.5 & 0.25 & 0.25 \\
0.25 & 0.5 & 0.25 \\
0.25 & 0.25 & 0.5
\end{array}\right)
$$

Therefore, a sample of $3^{T+1}$ possible scenarios is obtained as $\theta_{t}$ can take three different values. Table 2 reports the main properties of the optimal value function for different simulation lengths and temporal horizons. 

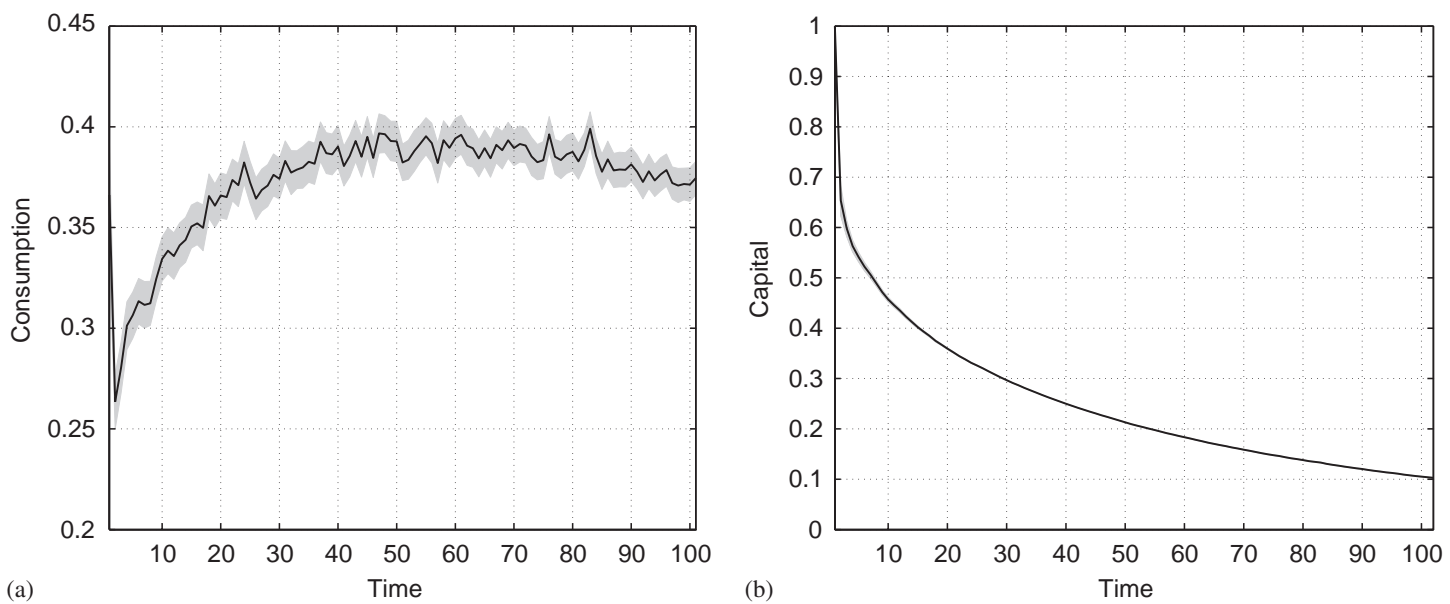

Fig. 5. Paths for $T=100$ and $|S|=100$.

It can be observed that, for $T=50$, a value of $|S|=50$ would have been considered enough for our purposes as

$$
\frac{\left|V_{25}^{*}-V_{50}^{*}\right|}{\left|V_{25}^{*}\right|}=0.0052
$$

which represents an error less than $1 \%$. Similarly, for $T=50$ and $|S|=100$ as

$$
\frac{\left|V_{50}^{*}-V_{100}^{*}\right|}{\left|V_{50}^{*}\right|}=0.0073
$$

which again represents an error less than $1 \%$.

In Fig. 5, the optimal paths for $E\left(c_{t}\right)$ and $E\left(k_{t}\right)$, respectively, are shown with their corresponding confidence intervals (at $95 \%$ level and with $T=100$ and $|S|=100$ ).

In Fig. 6, the optimal path for the normalized Euler equation errors is shown with their corresponding pointwise confidence intervals (at $95 \%$ level and with $T=100$ and $|S|=100$ ).

From Table 2 and Figs. 5, 6 it can be deduced that the proposed decomposition methodology obtains sufficiently accurate results for practical purposes. For instance, the accuracy obtained for this type of models by Borağan et al., in [11], is around $10^{-8}$ (though these authors consider another normalization of the Euler equation). Note that, with the proposed methodology, we obtain an accuracy around of $10^{-7}$ (with a termination tolerance for the subproblems of $\varepsilon=10^{-8}$ ). Moreover, the maximum error obtained by Borağan et al. is around $10^{-4}$ while the maximum error obtained by us is around $10^{-6}$ (corresponding to the upper level of the confidence interval, as shown in Fig. 6).

\section{An international model with uncertainty}

In this section, we consider a model with a finite number of heterogeneous agents. These models has been fruitfully applied to study many questions in international economics, for example the co-movement of output, investment and consumption across countries and international capital flows between countries (for a review, see for instance [30]). Because of its high dimension, the computational solution of this problem is a quite challenging task.

The formulation of this model is as follows. Suppose a dynamic economy with $N$ countries where each country is populated by a representative consumer, over a number of years, $t=0,1, \ldots$. A social planner maximizes a weighted sum of the expected lifetime utilities of the countries' representative consumers subject to the resource constraint. 


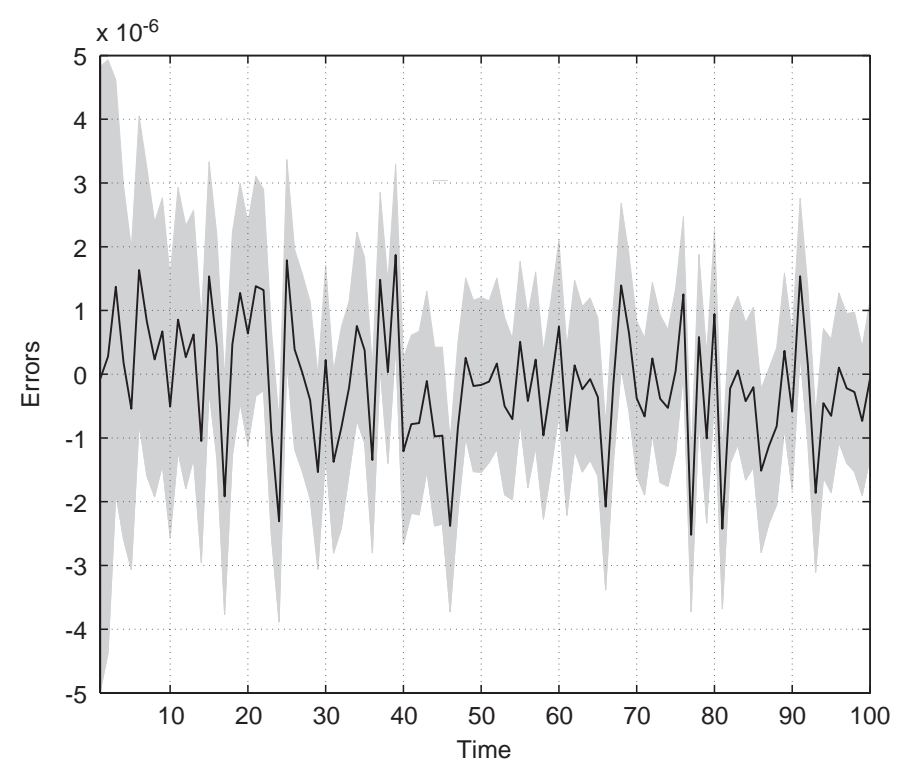

Fig. 6. Euler's error path (with confidence intervals) for $T=100$ and $|S|=100$.

The resulting model has the following form

$$
\begin{aligned}
\max & E\left[\sum_{n=1}^{N} \frac{1}{N} \sum_{t=0}^{\infty} \beta^{t} u\left(c_{t}^{n}\right)\right] \\
\text { s.t. } & k_{t+1}^{n}+\left(\frac{\varphi}{2}\left(k_{t+1}^{n}-k_{t}^{n}\right)^{\xi}\right) / k_{t}^{n}=i_{t}^{n}+(1-\delta) k_{t}^{n} \quad \text { for all } t \text { and } n, \\
& \sum_{n=1}^{N} c_{t}^{n}+\sum_{n=1}^{N} i_{t}^{n}=\sum_{n=1}^{N} A a_{t}^{n}\left(k_{t}^{n}\right)^{\alpha} \quad \text { for all } t,
\end{aligned}
$$

where $c_{t}^{n}, k_{t}^{n}, i_{t}^{n}$ denote consumption, capital and investment, respectively, at time $t$ and in country $n, \beta$ is the discount parameter, $\delta$ is the depreciation rate and $\varphi$ is the adjustment cost parameter. Finally, $A=(1-\beta+\beta \delta) / \alpha \beta$.

In this case, the stochastic process $a_{t}^{n}$ determining the technology shock is supposed to follow the law of motion:

$$
\ln a_{t}^{n}=\eta \ln a_{t-1}^{n}+\sigma\left(\varepsilon_{t}-\varepsilon_{t}^{n}\right), \quad n=1, \ldots, N, t=1, \ldots,
$$

where $\varepsilon_{t}$ and $\varepsilon_{t}^{n}$ are i.i.d. random variables with a standard Normal distribution. Note that the nonlinearity is measured by the parameter $\varphi$. When this parameter is greater than zero then the problem becomes strongly nonlinear. This is due to the fact that the term $\varphi / 2\left(k_{t+1}^{n}-k_{t}^{n}\right)^{\xi}$ in (13) corresponds to a penalization term controlled by the penalization parameter $\varphi$. For $\xi>1$, the constraint is nonlinear and nonconvex.

We have considered a utility function $u(c)=\left(c^{\rho}-1\right) / \rho$ with $\rho=0.4$. The parameters in the constraints are $\delta=0.025$, $\alpha=0.025$ and $\xi=2$. The discount factor is $\beta=0.99$ and the regularization parameter has been set to $\varepsilon_{T}=10^{-8}$. For the stochastic shock simulations we have selected $\eta=0.95$ and $\sigma=0.95$.

In this context, after choosing $T$ and representing the uncertainty by a scenario tree $S$, problem (13) can be broken into a set of independent problems for each $t$ and each $s \in S$. Therefore, we consider a decomposition of the problem by time and scenarios. At each iteration of the procedure, the following $|S|(T+1)$ subproblems are solved (Fig. 7).

Again, the solution of the subproblems SPt, for $t=0, \ldots, T$, have been solved using KNITRO with an active-set sequential linear-quadratic method. Note that the number of variables and constraints of the subproblems are $3 N$ and $N+1$, respectively. 
SP0. For $t=0$ :

$$
\begin{aligned}
& \max \sum_{n=1}^{N} \beta^{0} u\left(c_{0, s}^{n}\right) \\
& \text { subject to } \bar{k}_{1, s}^{n}+\left(\frac{\varphi}{2}\left(\bar{k}_{1, s}^{n}-\bar{k}_{0, s}^{n}\right)^{\xi}\right) / \bar{k}_{0, s}^{n}=i_{0, s}^{n}+(1-\delta) \bar{k}_{0, s}^{n}, \quad \text { for all } n \\
& \sum_{n=1}^{N} c_{0, s}^{n}+\sum_{n=1}^{N} i_{0, s}^{n}=\sum_{n=1}^{N} A a_{0, s}^{n}\left(\bar{k}_{0, s}^{n}\right)^{\alpha},
\end{aligned}
$$

(with $\bar{k}_{1, s}^{n}$-fixed from previous iteration);

SPt. For $t=1, \ldots, T-1$ :

$$
\begin{aligned}
& \max \quad \sum_{n=1}^{N}\left(\beta^{t} u\left(c_{t, s}^{n}\right)-\bar{\lambda}_{t-1, s}^{n}\left(k_{t, s}^{n}+\right.\right. \\
& \left.\left.\quad\left(\frac{\varphi}{2}\left(k_{t, s}^{n}-\bar{k}_{t-1, s}^{n}\right)^{\xi}\right) / \bar{k}_{t-1, s}^{n}-\bar{i}_{t-1, s}^{n}-(1-\delta) \bar{k}_{t-1, s}^{n}\right)\right) \\
& \text { subject to } \bar{k}_{t+1, s}^{n}+\left(\frac{\varphi}{2}\left(\bar{k}_{t+1, s}^{n}-k_{t, s}^{n}\right)^{\xi}\right) / k_{t, s}^{n}=i_{t, s}^{n}+(1-\delta) k_{t, s}^{n}, \quad \text { for all } n, \\
& \sum_{n=1}^{N} c_{t, s}^{n}+\sum_{n=1}^{N} i_{t, s}^{n}=\sum_{n=1}^{N} A a_{t, s}^{n}\left(k_{t, s}^{n}\right)^{\alpha},
\end{aligned}
$$

(with $\bar{\lambda}_{t-1, s}^{n}, \bar{k}_{t-1, s}^{n}, \bar{k}_{t+1, s}^{n}$ and $\bar{i}_{t-1, s}^{n}$-fixed from previous iteration);

SPT. For $t=T$ :

$$
\begin{aligned}
& \max \quad \sum_{n=1}^{N}\left(\beta^{T} u\left(c_{T, s}^{n}\right)-\bar{\lambda}_{T-1, s}^{n}\left(k_{T, s}^{n}+\right.\right. \\
& \left.\left.\quad\left(\frac{\varphi}{2}\left(k_{T, s}^{n}-\bar{k}_{T-1, s}^{n}\right)^{\xi}\right) / \bar{k}_{T-1, s}^{n}-\bar{i}_{T-1, s}^{n}-(1-\delta) \bar{k}_{T-1, s}^{n}\right)+\epsilon_{T} \ln \left(\bar{k}_{T+1, s}^{n}\right)\right) \\
& \text { subject to } \quad \bar{k}_{T+1, s}^{n}+\left(\frac{\varphi}{2}\left(\bar{k}_{T+1, s}^{n}-k_{T, s}^{n}\right)^{\xi}\right) k_{T, s}^{n}=i_{T, s}^{n}+(1-\delta) k_{T, s}^{n}, \quad \text { for all } n, \\
& \sum_{n=1}^{N} c_{T, s}^{n}+\sum_{n=1}^{N} i_{T, s}^{n}=\sum_{n=1}^{N} A a_{T, s}^{n}\left(k_{T, s}^{n}\right)^{\alpha},
\end{aligned}
$$

and compute $\bar{k}_{T+1, s}^{n}$ as a solution of: $k_{T+1, s}^{n}+\left(\frac{\varphi}{2}\left(k_{T+1, s}^{n}-k_{T, s}^{n}\right)^{\xi}\right) / k_{T, s}^{n}=i_{T, s}^{n}+(1-\delta) k_{T, s}^{n}$ (with $\bar{\lambda}_{T-1, s}^{n}, \bar{k}_{T-1, s}^{n}, \bar{k}_{T+1, s}^{n}$ and $\bar{i}_{T-1, s}^{n}$-fixed from previous iteration).

Fig. 7. Basic iteration of the decomposition algorithm in the international model with uncertainty.

Table 3 reports the computational time (in seconds) that the proposed decomposition methodology needs to find an optimal solution for a temporal horizon $T=100$ and $S=100$ replications. Several cases were run to study the computational time as a function of the number of countries and the nonlinearity of the problem. Table 3 also reports the confidence intervals of the optimal value function for the different number of countries and values of the parameter $\varphi$.

From Table 3 it can be deduced that the proposed decomposition methodology obtains sufficiently accurate results for practical purposes. Moreover, the required computed time to solve the problems is reasonable, taking into account the dimension of them. To the best of our knowledge, no results exist in the literature showing solutions of the presented model for $N>10$.

Attempts to solve this problem using parametric approximations are unsatisfactory. Krueger et al., in [31], introduce the Smolyak's algorithm to compute solutions of large-scale dynamic economic models. To document the performance of the method they consider this model with $N<4$ countries (and $\varphi \in[0,2]$ ). Its computing time to converge to the solution is 4 hours and 15 minutes for $N=4$ and $\varphi=0$, and 12 hours and 31 minutes for $N=4$ and $\varphi=2$.

Maliar and Maliar, in [32], describe a version of the simulation-based parameterized expectations algorithm (PEA), introduced in [6], that is only successful in finding the solutions to models with a number of countries $(N)$ less than 10 . For the sake of completeness, Table 4 provides a comparison between the computational time obtained in [32] (with a Pentium IV, $2.8 \mathrm{Ghz}$ ) and the corresponding time obtained by the proposed decomposition algorithm. We have used the 


\begin{tabular}{rccc}
\hline$N$ & $\varphi$ & CPU Time & Confidence interval $(95 \%)$ of $E\left[V^{*}\right]$ \\
\hline 2 & 0 & $2.35 \mathrm{e}+003$ & {$[-132.04,-131.59]$} \\
4 & 0 & $4.72 \mathrm{e}+003$ & {$[-132.54,-131.53]$} \\
10 & 0 & $9.97 \mathrm{e}+003$ & {$[-133.89,-132.27]$} \\
20 & 0 & $1.98 \mathrm{e}+004$ & {$[-135.72,-133.74]$} \\
2 & 2 & $4.05 \mathrm{e}+003$ & {$[-133.91,-133.11]$} \\
4 & 2 & $7.21 \mathrm{e}+003$ & {$[-134.61,-133.65]$} \\
10 & 2 & $1.19 \mathrm{e}+004$ & {$[-135.93,-134.49]$} \\
20 & 2 & $2.66 \mathrm{e}+004$ & {$[-136.47,-134.78]$} \\
2 & 10 & $6.03 \mathrm{e}+003$ & {$[-138.21,-137.20]$} \\
4 & 10 & $1.01 \mathrm{e}+004$ & {$[-139.06,-137.85]$} \\
20 & 10 & $1.53 \mathrm{e}+004$ & {$[-141.05,-139.32]$} \\
\hline
\end{tabular}

Table 4

CPU time comparison

\begin{tabular}{rllr}
\hline$N$ & $\varphi$ & CPU with PEA & CPU with decomposition \\
\hline 2 & 10 & $3.03 \mathrm{e}+002$ & $6.03 \mathrm{e}+003$ \\
4 & 10 & $8.40 \mathrm{e}+002$ & $1.01 \mathrm{e}+004$ \\
10 & 10 & $2.05 \mathrm{e}+004$ & $1.53 \mathrm{e}+004$ \\
20 & 10 & Not solved & $4.26 \mathrm{e}+004$ \\
\hline
\end{tabular}

same set of instances as in [32]. It can be observed that the proposed decomposition method outperforms the algorithm in [32] when the size of the problem increases.

\section{Conclusions and further extensions}

In this paper, we make a computational contribution for a rich toolbox of optimization models that economists use to analyze various facets of the economy. The solvability of these models suffers from the curse of dimensionality, which limits practitioners from the modelling standpoint. In this sense, we have introduced a novelty decomposition methodology for the computation of solutions of dynamic stochastic economics problems.

The proposed approach deflates the dimensionality of the models by breaking the problem into a set of smaller independent subproblems. We have shown the decomposition method works very well in practice, better than a direct method (when this is feasible).

We have solved several high-dimension problems. The numerical results have revealed the efficiency of the methodology in terms of computing time and accuracy, concluding that the proposed approach is promising for application in many economics problems with similar structure.

\section{Acknowledgments}

We thank Prof. A. Balbás and J. M. Vidal-Sanz for their helpful comments and suggestions, which have led to an improved version of this paper. This research has been partly supported by the European Commissiop through project FP6-2004-505509, the Ministerio de Educación y Ciencia of Spain, through projects MTM2004-02334 and SEJ2004-00672, and Comunidad Autónoma de Madrid, through the proyect s-0505/tic/000230. 


\section{References}

[1] Rust J. Numerical dynamic programming in economics. In: Amman H, Kendrick D, Rust J, editors. Handbook of computational economics. Amsterdam: North-Holland; 1996.

[2] Judd KL. Approximation, perturbation, and projection solution methods in economics. In: Amman H, Kendrick D, Rust J, editors. Handbook of computational economics. Amsterdam: North-Holland; 1996.

[3] Gaspar J, Judd KL. Solving large-scale rational-expectations models. Macroeconomic Dynamics 1997;1:45-75.

[4] Judd KL, Wang S-P. Solving a savings allocation problem by numerical dynamic programming with shape-preserving interpolation. Computers and Operations Research 2000;27(5):399-408.

[5] Wang S-P. Shape-preserving computation in economic growth models. Computers and Operations Research 2001;28(7):637-47.

[6] den Haan WJ, Marcet A. Solving the stochastic growth model by parameterizing expectations. Journal of Business and Economic Statistics 1990;8:31-4.

[7] Judd KL. Projection methods for solving aggregate growth models. Journal of Economic Theory 1992;58:410-52.

[8] Judd KL, Guu S-M. Perturbation solution methods for economic growth models. In: Economic and financial modelling with mathematica. New York: Springer; 1993.

[9] Taylor JB, Uhlig H. Solving nonlinear stochastic growth models: a comparison of alternative solution methods. Journal of Business Economic and Stastistics 1990;8:1-18.

[10] Judd KL, Kubler F, Schmedders K. Computational methods for dynamic equilibria with heterogeneous agents. In: Mathias Dewatripont J, Lars Peter Hansen, Stephen Turnovsky, editors. Advances in Economics and Econometrics. Cambridge: Cambridge University Press; 2003.

[11] Borağan S, Fernández-Villaverde J, Rubio-Ramírez JF. Comparing solution methods for dynamic equilibrium economies. Technical report, Federal Reserve Bank of Atlanta: 2004.

[12] Kraft D. On converting optimal control problems into nonlinear programming problems. Computational mathematical programming, NATO ASI Series F: Computer and Systems Science 1985;15:261-80.

[13] Bergounioux M, Ito K, Kunisch K. Primal-dual strategy for constrained optimal control problems. SIAM Journal of Control and Optimization 1997;35:1524-43.

[14] Dantzig GB, Wolfe P. Decomposition principle for linear programs. Operations Research 1960;8:101-11.

[15] Benders JF. Partioning procedures for solving mixed variables programming problems. Numerische Mathematik 1962;4:238-52.

[16] Geoffrion AM. Generalized Benders decomposition. Journal of Optimization Theory and Applications 1972;10(4):237-60.

[17] Cohen G, Miara B. Optimization with an auxiliary constraint and decomposition. SIAM Journal of Control and Optimization 1990;28(1): 137-57.

[18] Rockafellar RT, Wets RJ-B. A Lagrangian finite generation technique for solving linear-quadratic problems in stochastic programming. Mathematical Programming Study 1986;28:63-93.

[19] Ruszczynski A. On convergence of an augmented lagrangian decomposition method for sparse convex optimization. Mathematics of Operations Research 1995;20(3):634-56.

[20] Conejo AJ, Nogales FJ, Prieto FJ. A decomposition procedure based on approximate Newton directions. Mathematical Programming 2002;93(3):495-515.

[21] Helgason T, Wallace SW. Approximate scenario solutions in the progressive hedging algorithm. Annals of Operations Research 1991;31: 425-44.

[22] Chun BJ, Robinson SM. Scenario analysis via bundle decomposition. Annals of Operations Research 1995;56:39-63.

[23] Mansur A, Whalley J. A decomposition algorithm for general equilibrium computation with application to international trade models. Econometrica 1982;50(6):1547-57.

[24] Debreu G. Theory of value. New York: Wiley; 1950.

[25] Ljungqvist L, Sargent TJ. Recursive macroeconomic theory. Cambridge, MA: The MIT Press; 2000.

[26] Judd KL. Numerical methods in economics. Cambridge: The MIT Press; 1998.

[27] Stokey NL, Lucas RE. Recursive methods in economic dynamics. Cambridge, MA: Harvard University Press; 1989.

[28] Mas-Colell A, Whinston MD, Green JR. Microeconomic theory. New York: Oxford University Press; 1995.

[29] Gülpinar N, Rustem B, Settergren R. Optimization approaches to scenario tree generation. Journal of Economics Dynamics and Control 2004;28:1291-315.

[30] Backus D, Kehoe P, Kidland F. International business cycles, theory and evidence. In: Cooley T, editor. Frontiers of business cycle research. Princeton, NJ: Princeton University Press; 1995.

[31] Krueger D, Kubler F, Malin B. Computing stochastic dynamic economic models with a large number of state variables: A description and applications of Smolyak's method. Manuscript; 2003.

[32] Maliar L, Maliar S. Comparing numerical solutions of models with heterogeneous agents (Model A): a simulation-based parameterized expectations algorithm. Manuscript; 2004. 